International Mathematical Forum, 2, 2007, no. 4, 155 - 166

\title{
Regular Elements of Some Semigroups of Linear Transformations and Matrices
}

\author{
S. Nenthein and Y. Kemprasit \\ Department of Mathematics, Faculty of Science, \\ Chulalongkorn University, Bangkok 10330, Thailand \\ E-mail: Sansanee.N@student.chula.ac.th \\ Yupaporn.K@Chula.ac.th
}

\begin{abstract}
Let $V$ be a vector space over a field $F$ and $L_{F}(V)$ the semigroup, under composition, of all linear transformations $\alpha: V \rightarrow V$ and $M_{n}(F)$ the multiplicative semigroup of all $n \times n$ matrices over $F$. It is well-known that $L_{F}(V)$ and $M_{n}(F)$ are regular semigroups. If $W$ is a subspace of $V$, let $I_{F}(V, W)$ and $K_{F}(V, W)$ be the subsemigroups of $L_{F}(V)$ defined by $I_{F}(V, W)=\left\{\alpha \in L_{F}(V) \mid \operatorname{im} \alpha \subseteq W\right\}$ and $K_{F}(V, W)=\{\alpha \in$ $\left.L_{F}(V) \mid W \subseteq \operatorname{ker} \alpha\right\}$. The purpose of this paper is to characterize the regular elements of the semigroups $I_{F}(V, W)$ and $K_{F}(V, W)$. These characterizations are then applied to determine respectively the regular elements of the subsemigroups $C_{n}(F, k)$ and $R_{n}(F, k)$ of $M_{n}(F)$ where $C_{n}(F, k)=\left\{A \in M_{n}(F) \mid A_{i j}=0\right.$ for all $i, j \in\{1, \ldots, n\}$ and $\left.j>k\right\}$ and $R_{n}(F, k)=\left\{A \in M_{n}(F) \mid A_{i j}=0\right.$ for all $i, j \in\{1, \ldots, n\}$ and $i>$ $k\}$.
\end{abstract}

Mathematics Subject Classification: 20M17, 20M20

Keywords: Regular element of a semigroup, semigroup of linear transformations, matrix semigroup.

\section{Introduction}

An element $x$ of a semigroup $S$ is said to be regular if $x=x y x$ for some $y \in S$, and $S$ is called a regular semigroup if every element of $S$ is regular.

Throughout, let $F$ be a field, $V$ a vector space over $F$ and $L_{F}(V)$ the semigroup, under composition, of all linear transformations $\alpha: V \rightarrow V$. In this paper, the image of $v \in V$ under $\alpha \in L_{F}(V)$ is written by $v \alpha$. Denote by im $\alpha$ and ker $\alpha$ the image and the kernel of $\alpha$, respectively. Also, let $M_{n}(F)$ 
denote the multiplicative semigroup of all $n \times n$ matrices over $F$. It is wellknown that $M_{n}(F) \cong L_{F}(V)$ if $\operatorname{dim}_{F}(V)=n$ ([4], page 330) and $L_{F}(V)$ is a regular semigroup ([2], page 63). Hence $M_{n}(F)$ is also a regular semigroup. The entry of $A \in M_{n}(F)$ in the $i \underline{t h}$ row and $j \underline{t h}$ column will be denoted by $A_{i j}$.

In 1952, Malcev [5] showed that every automorphism of $T(X)$, the full transformation semigroup on a set $X$, is inner. Symons [7] generalized this result in 1975 by considering the subsemigroup $T(X, Y), \emptyset \neq Y \subseteq X$, of $T(X)$ consisting of all $\alpha \in T(X)$ whose images are contained in $Y$, that is,

$$
T(X, Y)=\{\alpha \in T(X) \mid \text { im } \alpha \subseteq Y\} .
$$

In [6], the authors gave a characterization of the regular elements of $T(X, Y)$. They also determined the number of regular elements in $T(X, Y)$ for a finite set $X$. It was given in terms of $|X|,|Y|$ and their related Stirling numbers. In this paper, we consider the subsemigroup $I_{F}(V, W)$ of $L_{F}(V)$ analogous to $T(X, Y)$ defined by

$$
I_{F}(V, W)=\left\{\alpha \in L_{F}(V) \mid \operatorname{im} \alpha \subseteq W\right\}
$$

where $W$ is a subspace of $V$. Its regular elements are characterized in this paper. The semigroup $I_{F}(V, W)$ motivates us to consider the subsemigroup $K_{F}(V, W)$ of $L_{F}(V)$ defined by

$$
K_{F}(V, W)=\left\{\alpha \in L_{F}(V) \mid W \subseteq \operatorname{ker} \alpha\right\}
$$

and its regular elements are also characterized. Notice that $I_{F}(V, W)=\{\alpha \in$ $\left.L_{F}(V) \mid V \alpha \subseteq W\right\}, K_{F}(V, W)=\left\{\alpha \in L_{F}(V) \mid W \alpha=\{0\}\right\}, I_{F}(V, V)=$ $L_{F}(V)=K_{F}(V,\{0\})$ and $I_{F}(V,\{0\})=\{0\}=K_{F}(V, V)$. In general, if $\{0\} \neq$ $W \subsetneq V$, then $I_{F}(V, W)$ and $K_{F}(V, W)$ are not subsets of each other. To see this, assume that $\{0\} \neq W \subsetneq V$. Let $B_{1}$ be a basis of $W$ and $B$ a basis of $V$ containing $B_{1}$. Define $\alpha, \beta \in L_{F}(V)$ on $B$ by bracket notation as follows:

$$
\alpha=\left[\begin{array}{cc}
v & B \backslash B_{1} \\
v & 0
\end{array}\right]_{v \in B_{1}}, \beta=\left[\begin{array}{cc}
B_{1} & v \\
0 & v
\end{array}\right]_{v \in B \backslash B_{1}} .
$$

Then $\operatorname{im} \alpha=\left\langle B_{1}\right\rangle=\operatorname{ker} \beta, \operatorname{ker} \alpha=\left\langle B \backslash B_{1}\right\rangle=\operatorname{im} \beta$. Therefore we deduce that $\alpha \in I_{F}(V, W) \backslash K_{F}(V, W)$ and $\beta \in K_{F}(V, W) \backslash I_{F}(V, W)$.

Our characterizations of the regular elements of $I_{F}(V, W)$ and $K_{F}(V, W)$ are then applied to determine respectively the regular elements of the matrix semigroups $C_{n}(F, k)$ and $R_{n}(F, k)$ where $1 \leq k \leq n$,

$$
\begin{aligned}
& C_{n}(F, k)=\left\{A \in M_{n}(F) \mid A_{i j}=0 \text { for all } i, j \in\{1, \ldots, n\} \text { and } j>k\right\}, \\
& R_{n}(F, k)=\left\{A \in M_{n}(F) \mid A_{i j}=0 \text { for all } i, j \in\{1, \ldots, n\} \text { and } i>k\right\} .
\end{aligned}
$$


In other words, $C_{n}(F, k)$ consists of all matrices in $M_{n}(F)$ of the form

$$
\left[\begin{array}{cccccc}
a_{11} & \cdots & a_{1 k} & 0 & \cdots & 0 \\
a_{21} & \cdots & a_{2 k} & 0 & \cdots & 0 \\
\vdots & & \vdots & \vdots & & \vdots \\
a_{n 1} & \cdots & a_{n k} & 0 & \cdots & 0
\end{array}\right]
$$

and $R_{n}(F, k)$ consists of all matrices in $M_{n}(F)$ of the form

$$
\left[\begin{array}{cccc}
a_{11} & a_{12} & \cdots & a_{1 n} \\
\vdots & \vdots & & \vdots \\
a_{k 1} & a_{k 2} & \cdots & a_{k n} \\
0 & 0 & \cdots & 0 \\
\vdots & \vdots & & \vdots \\
0 & 0 & \cdots & 0
\end{array}\right] .
$$

Observe that $R_{n}(F, n)=M_{n}(F)=C_{n}(F, n)$. It is clearly seen that if $t_{1}, \ldots, t_{k} \in$ $\{1, \ldots, n\}$ with $t_{1}<t_{2}<\cdots<t_{k}$, then $S_{1}$ and $S_{2}$ defined by

$$
\begin{aligned}
& S_{1}=\left\{A \in M_{n}(F) \mid A_{i j}=0 \text { for all } i, j \in\{1, \ldots, n\} \text { and } j \notin\left\{t_{1}, \ldots, t_{k}\right\}\right\}, \\
& S_{2}=\left\{A \in M_{n}(F) \mid A_{i j}=0 \text { for all } i, j \in\{1, \ldots, n\} \text { and } i \notin\left\{t_{1}, \ldots, t_{k}\right\}\right\}
\end{aligned}
$$

are subsemigroups of $M_{n}(F)$ which are clearly isomorphic to $C_{n}(F, k)$ and $R_{n}(F, k)$, respectively.

Throughout, let $W$ be a subspace of $V$ and $k \in\{1, \ldots, n\}$.

\section{The Semigroups $I_{F}(V, W)$ and $K_{F}(V, W)$}

First, we recall the basic fact of linear transformations which will be often used. If $\alpha \in L_{F}(V), B_{1}$ is a basis of $\operatorname{ker} \alpha, B_{2}$ is a basis of im $\alpha$ and for each $u \in B_{2}$, choose an element $u^{\prime} \in V$ such that $u^{\prime} \alpha=u$, then $B_{1} \cup\left\{u^{\prime} \mid u \in B_{2}\right\}$ is a basis of $V$.

Theorem 2.1. For $\alpha \in I_{F}(V, W), \alpha$ is a regular element of $I_{F}(V, W)$ if and only if im $\alpha=W \alpha$.

Proof. If $\alpha=\alpha \beta \alpha$ for some $\beta \in I_{F}(V, W)$, then $W \alpha \subseteq V \alpha=V \alpha \beta \alpha=$ $(V \alpha \beta) \alpha \subseteq W \alpha$, so im $\alpha=W \alpha$.

For the converse, assume that im $\alpha=W \alpha$. Let $B_{1}$ be a basis of $\operatorname{ker} \alpha, B_{2}$ a basis of im $\alpha$ and $B_{3}$ a basis of $V$ containing $B_{2}$. Since im $\alpha=W \alpha$, for each element $u \in B_{2}$, there is an element $u^{\prime} \in W$ such that $u^{\prime} \alpha=u$. Then $B_{1} \cup\left\{u^{\prime} \mid u \in B_{2}\right\}$ is a basis of $V$. Define $\beta \in L_{F}(V)$ on the basis $B_{3}$ of $V$ by

$$
\beta=\left[\begin{array}{cc}
u & B_{3} \backslash B_{2} \\
u^{\prime} & 0
\end{array}\right]_{u \in B_{2}} .
$$


Then $\operatorname{im} \beta=\left\langle\left\{u^{\prime} \mid u \in B_{2}\right\}\right\rangle \subseteq W$, so $\beta \in I_{F}(V, W)$. Since $B_{1} \alpha \beta \alpha=\{0\}=$ $B_{1} \alpha$ and $u^{\prime} \alpha \beta \alpha=u \beta \alpha=u^{\prime} \alpha$ for all $u \in B_{2}$, we have that $\alpha=\alpha \beta \alpha$. Hence $\alpha$ is a regular element of $I_{F}(V, W)$, as desired.

Corollary 2.2. The semigroup $I_{F}(V, W)$ is regular if and only if either $W=V$ or $W=\{0\}$.

Proof. Assume that $\{0\} \subsetneq W \subsetneq V$. Let $B_{1}$ be a basis of $W$ and $B$ a basis of $V$ containing $B_{1}$. Let $w \in B_{1}$ and define $\alpha \in L_{F}(V)$ by

$$
\alpha=\left[\begin{array}{cc}
B_{1} & B \backslash B_{1} \\
0 & w
\end{array}\right] .
$$

Then $\operatorname{im} \alpha=\langle w\rangle \subseteq W$ and $W \alpha=\left\langle B_{1}\right\rangle \alpha=\{0\}$, thus im $\alpha \neq W \alpha$. Hence $\alpha \in I_{F}(V, W)$ and by Theorem 2.1, $\alpha$ is not a regular element of $I_{F}(V, W)$.

Since $I_{F}(V, V)=L_{F}(V)$ and $I_{F}(V,\{0\})=\{0\}$, the converse holds.

Theorem 2.3. For $\alpha \in K_{F}(V, W), \alpha$ is a regular element of $K_{F}(V, W)$ if and only if im $\alpha \cap W=\{0\}$.

Proof. Assume that $\alpha=\alpha \beta \alpha$ for some $\beta \in K_{F}(V, W)$. If $v \in \operatorname{im} \alpha \cap W$, then $v \in W$ and $v=u \alpha$ for some $u \in V$, and hence $v=u \alpha=u \alpha \beta \alpha=v \beta \alpha \in$ $W \beta \alpha=\{0\}$. This shows that im $\alpha \cap W=\{0\}$.

Conversely, assume that $\operatorname{im} \alpha \cap W=\{0\}$. Let $B_{1}$ be a basis of $\operatorname{ker} \alpha, B_{2}$ a basis of im $\alpha$ and $B_{3}$ a basis of $W$. Since im $\alpha \cap W=\{0\}, B_{2} \cap B_{3}=\emptyset$ and $B_{2} \cup B_{3}$ is a basis of im $\alpha+W$. Let $B_{4}$ be a basis of $V$ containing $B_{2} \cup B_{3}$. For each element $u \in B_{2}$, let $u^{\prime} \in V$ be such that $u^{\prime} \alpha=u$. Then $B_{1} \cup\left\{u^{\prime} \mid u \in B_{2}\right\}$ is a basis of $V$. Define $\beta \in L_{F}(V)$ by

$$
\beta=\left[\begin{array}{cc}
u & B_{4} \backslash B_{2} \\
u^{\prime} & 0
\end{array}\right]_{u \in B_{2}} .
$$

Since $B_{3} \subseteq B_{4} \backslash B_{2}$, it follows that $W \beta=\left\langle B_{3}\right\rangle \beta=\{0\}$, so $\beta \in K_{F}(V, W)$. Moreover, $B_{1} \alpha \beta \alpha=\{0\}=B_{1} \alpha$ and $u^{\prime} \alpha \beta \alpha=u \beta \alpha=u^{\prime} \alpha$ for all $u \in B_{2}$. Hence we have $\alpha=\alpha \beta \alpha$, so $\alpha$ is a regular element of $K_{F}(V, W)$.

Corollary 2.4. The semigroup $K_{F}(V, W)$ is regular if and only if either $W=V$ or $W=\{0\}$.

Proof. Assume that $\{0\} \subsetneq W \subsetneq V$. Let $B_{1}, B, w$ and $\alpha \in L_{F}(V)$ be as in Corollary 2.2. Since $W \alpha=\{0\}$, we have $\alpha \in K_{F}(V, W)$. Also, im $\alpha \cap W=$ $\langle w\rangle \cap W=\langle w\rangle \neq\{0\}$. By Theorem 2.3, $\alpha$ is not a regular element of $K_{F}(V, W)$.

The converse holds since $K_{F}(V, V)=\{0\}$ and $K_{F}(V,\{0\})=L_{F}(V)$. 


\section{The Matrix Semigroups $C_{n}(F, k)$ and $R_{n}(F, k)$}

To characterize the regular elements of $C_{n}(F, k)$ and $R_{n}(F, k)$ by Theorem 2.1 and Theorem 2.3, respectively, some lemmas are needed.

For convenience, following [2], the set of all regular elements of a semigroup $S$ may be denoted by $\operatorname{Reg}(S)$.

For a vector space $V$, let $V^{*}$ and $V^{* *}$ be the dual space and the double dual space of $V$, respectively. For $A \subseteq V$, the annihilator of $A$ is denoted by $A^{0}$, that is,

$$
A^{0}=\left\{f \in V^{*} \mid f(v)=0 \text { for all } v \in A\right\}
$$

and let $A^{00}=\left(A^{0}\right)^{0}$, that is,

$$
A^{00}=\left\{T \in V^{* *} \mid T(f)=0 \text { for all } f \in A^{0}\right\} .
$$

For $\left(x_{1}, \ldots, x_{n}\right) \in F^{n}$, define $h_{\left(x_{1}, \ldots, x_{n}\right)}: F^{n} \rightarrow F$ by

$$
h_{\left(x_{1}, \ldots, x_{n}\right)}\left(t_{1}, \ldots, t_{n}\right)=t_{1} x_{1}+\cdots+t_{n} x_{n} \text { for all }\left(t_{1}, \ldots, t_{n}\right) \in F^{n} \text {. }
$$

Then we have

$$
\left(F^{n}\right)^{*}=\left\{h_{\left(x_{1}, \ldots, x_{n}\right)} \mid\left(x_{1}, \ldots, x_{n}\right) \in F^{n}\right\}
$$

([1], page 149). For $x \in F^{n}$, define $T_{x}:\left(F^{n}\right)^{*} \rightarrow F$ by

$$
T_{x}(f)=f(x) \text { for all } x \in F^{n} \text {. }
$$

Then

$$
\begin{aligned}
& \left(F^{n}\right)^{* *}=\left\{T_{x} \mid x \in F^{n}\right\} \quad \text { and } \\
& T_{x} \neq T_{y} \text { for all distinct } x, y \in F^{n}
\end{aligned}
$$

([1], page 147). If $U$ is a subspace of $F^{n}$, then

$$
U^{00}=\left\{T_{u} \mid u \in U\right\}
$$

([1], page 148-149). Note that if $A_{1}$ and $A_{2}$ are subsets of $F^{n}$ such that $A_{1} \subseteq A_{2}$, then $A_{1}^{0} \supseteq A_{2}^{0}$ and $A_{1}^{00} \subseteq A_{2}^{00}$.

Lemma 3.1. Let $\left(a_{11}, \ldots, a_{1 n}\right), \ldots,\left(a_{m 1}, \ldots, a_{m n}\right),\left(b_{1}, \ldots, b_{n}\right)$ be elements of $F^{n}$. Then the following two conditions are equivalent.

(i) $\left(b_{1}, \ldots, b_{n}\right) \in\left\langle\left(a_{11}, \ldots, a_{1 n}\right), \ldots,\left(a_{m 1}, \ldots, a_{m n}\right)\right\rangle$.

(ii) For every $\left(x_{1}, \ldots, x_{n}\right) \in F^{n}, a_{i 1} x_{1}+\cdots+a_{i n} x_{n}=0$ for all $i \in\{1, \ldots, m\}$, then $b_{1} x_{1}+\cdots+b_{n} x_{n}=0$. 
Proof. Let $U_{1}=\left\langle\left(a_{11}, \ldots, a_{1 n}\right), \ldots,\left(a_{m 1}, \ldots, a_{m n}\right)\right\rangle$ and $U_{2}=\left\langle\left(b_{1}, \ldots, b_{n}\right)\right\rangle$.

Assume that (i) holds. Then $U_{2} \subseteq U_{1}$ which imlpies that $U_{2}^{0} \supseteq U_{1}^{0}$. Let $\left(x_{1}, \ldots, x_{n}\right) \in F^{n}$ be such that $a_{i 1} x_{1}+\cdots+a_{i n} x_{n}=0$ for all $i \in\{1, \ldots, m\}$. Then

$$
h_{\left(x_{1}, \ldots, x_{n}\right)}\left(a_{i 1}, \ldots, a_{i n}\right)=0 \text { for all } i \in\{1, \ldots, m\} .
$$

It follows that $h_{\left(x_{1}, \ldots, x_{n}\right)} \in U_{1}^{0}$. But $U_{1}^{0} \subseteq U_{2}^{0}$, so $h_{\left(x_{1}, \ldots, x_{n}\right)}\left(b_{1}, \ldots, b_{n}\right)=0$, that is, $b_{1} x_{1}+\cdots+b_{n} x_{n}=0$. Hence (ii) holds.

To show that (ii) implies (i), assume that (ii) holds. Then we have that for every $\left(x_{1}, \ldots, x_{n}\right) \in F^{n}, h_{\left(x_{1}, \ldots, x_{n}\right)} \in\left\langle\left\{\left(a_{11}, \ldots, a_{1 n}\right), \ldots,\left(a_{m 1}, \ldots, a_{m n}\right)\right\}\right\rangle^{0}$ implies that $h_{\left(x_{1}, \ldots, x_{n}\right)} \in\left\langle\left\{\left(b_{1}, \ldots, b_{n}\right)\right\}\right\rangle^{0}$. This follows from (1) that $U_{1}^{0} \subseteq U_{2}^{0}$. Then $U_{2}^{00} \subseteq U_{1}^{00}$. Hence by (3),

$$
\left\{T_{x} \mid x \in U_{2}\right\}=U_{2}^{00} \subseteq U_{1}^{00}=\left\{T_{x} \mid x \in U_{1}\right\} .
$$

By (2), we deduce that $U_{2} \subseteq U_{1}$, so (i) holds.

For a matrix $A \in M_{n}(F)$, define $g_{A}: F^{n} \rightarrow F^{n}$ by

$$
x g_{A}=x A \text { for all } x \in F^{n} .
$$

Clearly, $g_{A} \in L_{F}\left(F^{n}\right)$ for all $A \in M_{n}(F)$. Let $\left\{e_{1}, \ldots, e_{n}\right\}$ be the standard basis of $F^{n}$ over $F$. Therefore we have

$$
e_{i} g_{A}=\left(A_{i 1}, \ldots, A_{\text {in }}\right) \text { for all } i \in\{1, \ldots, n\} \text { and } A \in M_{n}(F) .
$$

Lemma 3.2. The mapping $\varphi: M_{n}(F) \rightarrow L_{F}\left(F^{n}\right)$ defined by $A \varphi=g_{A}$ for all $A \in M_{n}(F)$ is an isomorphism from $M_{n}(F)$ onto $L_{F}\left(F^{n}\right)$.

Proof. It is clear that $\varphi$ is a homomorphism. It follows from (4) that $\varphi$ is 1-1. If $\alpha \in L_{F}\left(F^{n}\right)$, then define $A \in M_{n}(F)$ by

$$
\left(A_{i 1}, \ldots, A_{i n}\right)=e_{i} \alpha \text { for all } i \in\{1, \ldots, n\} .
$$

Then by (4), $e_{i} g_{A}=e_{i} \alpha$ for all $i \in\{1, \ldots, n\}$, and thus $A \varphi=g_{A}=\alpha$. Hence the lemma is proved.

Lemma 3.3. Let $U_{1}$ and $U_{2}$ be subspaces of $F^{n}$ spanned by $\left\{e_{1}, \ldots, e_{k}\right\}$ and $\left\{e_{k+1}, \ldots, e_{n}\right\}$, respectively. Then

(i) $I_{F}\left(F^{n}, U_{1}\right)=\left\{g_{A} \mid A \in C_{n}(F, k)\right\}$ and

(ii) $K_{F}\left(F^{n}, U_{2}\right)=\left\{g_{A} \mid A \in R_{n}(F, k)\right\}$. 
Proof. We have from the definitions of $U_{1}$ and $U_{2}$ that

$$
U_{1}=\left\{\left(x_{1}, \ldots, x_{k}, 0, \ldots, 0\right) \mid x_{1}, \ldots, x_{k} \in F\right\}
$$

and

$$
U_{2}= \begin{cases}\{(0, \ldots, 0)\} & \text { if } k=n \\ \left\{\left(0, \ldots, 0, x_{k+1}, \ldots, x_{n}\right) \mid x_{k+1}, \ldots, x_{n} \in F\right\} & \text { if } k<n\end{cases}
$$

(i) For $A \in M_{n}(F)$,

$$
\begin{aligned}
g_{A} \in I_{F}\left(F^{n}, U_{1}\right) & \Leftrightarrow \operatorname{im} g_{A} \subseteq U_{1} \\
& \Leftrightarrow\left(A_{i 1}, \ldots, A_{i n}\right) \in U_{1} \text { for all } i \in\{1, \ldots, n\} \text { from }(4) \\
& \Leftrightarrow A_{i j}=0 \text { for all } i, j \in\{1, \ldots, n\} \text { with } j>k \\
& \Leftrightarrow A \in C_{n}(F, k) .
\end{aligned}
$$

Hence by Lemma 3.2, (i) holds.

(ii) If $k=n$, then $K_{F}\left(F^{n}, U_{2}\right)=L_{F}\left(F^{n}\right)$ and $R_{n}(F, k)=M_{n}(F)$, so (ii) holds by Lemma 3.2. Next, assume that $k<n$. Then for $A \in M_{n}(F)$,

$$
\begin{aligned}
g_{A} \in K_{F}\left(F^{n}, U_{2}\right) & \Leftrightarrow U_{2} \subseteq \operatorname{ker} g_{A} \\
& \Leftrightarrow U_{2} g_{A}=\{(0, \ldots, 0)\} \\
& \Leftrightarrow e_{i} g_{A}=(0, \ldots, 0) \text { for all } i \in\{k+1, \ldots, n\} \\
& \Leftrightarrow\left(A_{i 1}, \ldots, A_{i n}\right)=(0, \ldots, 0) \\
& \Leftrightarrow A \in R_{n}(F, k) .
\end{aligned}
$$

Hence (ii) holds by Lemma 3.2.

Theorem 3.4. For $A \in C_{n}(F, k), A$ is regular in $C_{n}(F, k)$ if and only if for any $x_{1}, \ldots, x_{k} \in F$,

$$
\begin{aligned}
A_{i 1} x_{1}+\cdots+ & A_{i k} x_{k}=0 \text { for all } i \in\{1, \ldots, k\} \\
& \Rightarrow A_{i 1} x_{1}+\cdots+A_{i k} x_{k}=0 \text { for all } i \in\{k+1, \ldots, n\},
\end{aligned}
$$

that is, for any $\left(x_{1}, \ldots, x_{k}\right) \in F^{k}$,

$$
\left[\begin{array}{ccc}
A_{11} & \cdots & A_{1 k} \\
\vdots & \ddots & \vdots \\
A_{k 1} & \cdots & A_{k k}
\end{array}\right]\left[\begin{array}{c}
x_{1} \\
\vdots \\
x_{k}
\end{array}\right]=\left[\begin{array}{c}
0 \\
\vdots \\
0
\end{array}\right] \Rightarrow\left[\begin{array}{ccc}
A_{k+1,1} & \cdots & A_{k+1, k} \\
\vdots & \ddots & \vdots \\
A_{n 1} & \cdots & A_{n k}
\end{array}\right]\left[\begin{array}{c}
x_{1} \\
\vdots \\
x_{k}
\end{array}\right]=\left[\begin{array}{c}
0 \\
\vdots \\
0
\end{array}\right] .
$$

Proof. Let $U$ be the subspace of $F^{n}$ spanned by $\left\{e_{1}, \ldots, e_{k}\right\}$. Then by Lemma 3.2 and Lemma 3.3(i), $C_{n}(F, k) \cong I_{F}\left(F^{n}, U\right)$ through the mapping $A \mapsto g_{A}$. 
Let $A \in C_{n}(F, k)$. Since $A_{i j}=0$ for all $i, j \in\{1, \ldots, n\}$ with $j>k$, by (4), we have

$$
\begin{aligned}
& \operatorname{im} g_{A}=\left\langle\left(A_{11}, \ldots, A_{1 k}, 0, \ldots, 0\right), \ldots,\left(A_{n 1}, \ldots, A_{n k}, 0, \ldots, 0\right)\right\rangle, \\
& U g_{A}=\left\langle\left(A_{11}, \ldots, A_{1 k}, 0, \ldots, 0\right), \ldots,\left(A_{k 1}, \ldots, A_{k k}, 0, \ldots, 0\right)\right\rangle .
\end{aligned}
$$

Hence

$$
\begin{aligned}
A \in \operatorname{Reg}\left(C_{n}(F, k)\right) \Leftrightarrow & g_{A} \in \operatorname{Reg}\left(I_{F}\left(F^{n}, U\right)\right) \\
\Leftrightarrow & \operatorname{im} g_{A}=U g_{A} \text { from Theorem } 2.1 \\
\Leftrightarrow & \left(A_{i 1}, \ldots, A_{i k}, 0, \ldots, 0\right) \\
& \quad \in \quad\left\langle\left(A_{11}, \ldots, A_{1 k}, 0, \ldots, 0\right), \ldots,\left(A_{k 1}, \ldots, A_{k k}, 0, \ldots, 0\right)\right\rangle \\
& \quad \text { for all } i \in\{k+1, \ldots, n\} \text { from }(6) \\
\Leftrightarrow & \left(A_{i 1}, \ldots, A_{i k}\right) \in\left\langle\left(A_{11}, \ldots, A_{1 k}\right), \ldots,\left(A_{k 1}, \ldots, A_{k k}\right)\right\rangle \\
\Leftrightarrow & \quad \text { for all } i \in\{k+1, \ldots, n\} \\
\Leftrightarrow & (5) \text { holds } \quad \text { from Lemma } 3.1 .
\end{aligned}
$$

Therefore the theorem is proved.

The following two corollaries are direct consequences of Theorem 3.4.

Corollary 3.5. If $A \in C_{n}(F, k)$ is of the form

$$
\left[\begin{array}{cccccc}
a_{11} & \cdots & a_{1 k} & 0 & \cdots & 0 \\
\vdots & \ddots & \vdots & \vdots & \ddots & \vdots \\
a_{k 1} & \cdots & a_{k k} & 0 & \cdots & 0 \\
0 & \cdots & 0 & 0 & \cdots & 0 \\
\vdots & \ddots & \vdots & \vdots & \ddots & \vdots \\
0 & \cdots & 0 & 0 & \cdots & 0
\end{array}\right]
$$

then $A$ is regular in $C_{n}(F, k)$.

We note here that if $S$ consists of all matrices $A \in M_{n}(F)$ of the form (7), then $S$ is a subsemigroup of $M_{n}(F)$ contained in $C_{n}(F, k)$ and $S \cong M_{k}(F)$. This implies that $S$ is a regular subsemigroup of $C_{n}(F, k)$.

Corollary 3.6. Let $k<n$ and $A \in C_{n}(F, k)$ be of the form

$$
\left[\begin{array}{cccccc}
0 & \cdots & 0 & 0 & \cdots & 0 \\
\vdots & \ddots & \vdots & \vdots & \ddots & \vdots \\
0 & \cdots & 0 & 0 & \cdots & 0 \\
a_{k+1,1} & \cdots & a_{k+1, k} & 0 & \cdots & 0 \\
\vdots & \ddots & \vdots & \vdots & \ddots & \vdots \\
a_{n 1} & \cdots & a_{n k} & 0 & \cdots & 0
\end{array}\right] .
$$

Then $A$ is regular in $C_{n}(F, k)$ if and only if $A$ is a zero matrix. 
Also, as a consequence of Theorem $3.4, C_{n}(F, k)$ is a regular semigroup only the case that $k=n$, or equivalently, $C_{n}(F, k)=M_{n}(F)$.

Corollary 3.7. The semigroup $C_{n}(F, k)$ is a regular semigroup if and only if $k=n$.

Proof. Assume that $k<n$. Define $A \in M_{n}(F)$ by

$$
A=\left[\begin{array}{cccc}
0 & 0 & \cdots & 0 \\
\vdots & \vdots & \ddots & 0 \\
0 & 0 & \cdots & 0 \\
1 & 0 & \cdots & 0
\end{array}\right] .
$$

Then $A \in C_{n}(F, k)$. Since $k<n$, by Corollary 3.6, $A$ is not regular in $C_{n}(F, k)$.

Since $C_{n}(F, n)=M_{n}(F)$, the converse holds.

Theorem 3.8. For $A \in R_{n}(F, k), A$ is regular in $R_{n}(F, k)$ if and only if for any $x_{1}, \ldots, x_{k} \in F$,

$$
\begin{aligned}
A_{1 j} x_{1}+\cdots+ & A_{k j} x_{k}=0 \text { for all } j \in\{1, \ldots, k\} \\
& \Rightarrow A_{1 j} x_{1}+\cdots+A_{k j} x_{k}=0 \text { for all } j \in\{k+1, \ldots, n\},
\end{aligned}
$$

that is, for any $\left(x_{1}, \ldots, x_{k}\right) \in F^{k}$,

$$
\begin{gathered}
{\left[\begin{array}{lll}
x_{1} & \cdots & x_{k}
\end{array}\right]\left[\begin{array}{ccc}
A_{11} & \cdots & A_{1 k} \\
\vdots & \ddots & \vdots \\
A_{k 1} & \cdots & A_{k k}
\end{array}\right]=\left[\begin{array}{lll}
0 & \cdots & 0
\end{array}\right]} \\
\Rightarrow\left[\begin{array}{lll}
x_{1} & \cdots & x_{k}
\end{array}\right]\left[\begin{array}{ccc}
A_{1, k+1} & \cdots & A_{1 n} \\
\vdots & \ddots & \vdots \\
A_{k, k+1} & \cdots & A_{k n}
\end{array}\right]=\left[\begin{array}{lll}
0 & \cdots & 0
\end{array}\right] .
\end{gathered}
$$

Proof. This is true if $k=n$ since $R_{n}(F, n)=M_{n}(F)$. Assume that $k<n$ and $U$ is a subspace of $F^{n}$ spanned by $\left\{e_{k+1}, \ldots, e_{n}\right\}$. By Lemma 3.2 and Lemma $3.3(\mathrm{ii}), R_{n}(F, k) \cong K_{F}\left(F^{n}, U\right)$ by $A \mapsto g_{A}$. Note that

$$
U=\left\{\left(0, \ldots, 0, x_{k+1}, \ldots, x_{n}\right) \mid x_{k+1}, \ldots, x_{n} \in F\right\} .
$$

Let $A \in R_{n}(F, k)$. Then $A_{i j}=0$ for all $i, j \in\{1, \ldots, n\}$ with $i>k$ and

$$
\begin{aligned}
A \in \operatorname{Reg}\left(R_{n}(F, k)\right) & \Leftrightarrow g_{A} \in \operatorname{Reg}\left(K_{F}\left(F^{n}, U\right)\right) \\
& \Leftrightarrow \operatorname{im} g_{A} \cap U=\{(0, \ldots, 0)\} \text { from Theorem 2.3. }
\end{aligned}
$$

Thus to prove the theorem, it suffices to show that im $g_{A} \cap U=\{(0, \ldots, 0)\}$ if and only if (8) holds. First, assume that im $g_{A} \cap U=\{(0, \ldots, 0)\}$ and let 
$x_{1}, \ldots, x_{k} \in F$ be such that $A_{1 j} x_{1}+\cdots+A_{k j} x_{k}=0$ for all $j \in\{1, \ldots, k\}$. Then

$$
\begin{aligned}
\left(x_{1}, \ldots\right. & \left., x_{k}, 0, \ldots, 0\right) g_{A} \\
& =\left(x_{1}, \ldots, x_{k}, 0, \ldots, 0\right) A \\
& =\left(A_{11} x_{1}+\cdots+A_{k 1} x_{k}, \ldots, A_{1 n} x_{1}+\cdots+A_{k n} x_{k}\right) \\
& =\left(0, \ldots, 0, A_{1, k+1} x_{1}+\cdots+A_{k, k+1} x_{k}, \ldots, A_{1 n} x_{1}+\cdots+A_{k n} x_{k}\right) \\
& \in \operatorname{im} g_{A} \cap U=\{(0, \ldots, 0)\} \quad \text { from }(9) .
\end{aligned}
$$

This implies that $A_{1 j} x_{1}+\cdots+A_{k j} x_{k}=0$ for all $j \in\{k+1, \ldots, n\}$.

Conversely, assume that (8) holds. Let $\left(y_{1}, \ldots, y_{n}\right) \in \operatorname{im} g_{A} \cap U$. Then $y_{j}=0$ for all $j \in\{1, \ldots, k\}$ by $(9)$ and $\left(y_{1}, \ldots, y_{n}\right)=\left(a_{1}, \ldots, a_{n}\right) g_{A}$ for some $\left(a_{1}, \ldots, a_{n}\right) \in F^{n}$. It follows that $A_{1 j} a_{1}+\cdots+A_{k j} a_{k}=y_{j}$ for all $j \in\{1, \ldots, n\}$. Then $A_{1 j} a_{1}+\cdots+A_{k j} a_{k}=0$ for all $j \in\{1, \ldots, k\}$. By (8), we have $A_{1 j} a_{1}+\cdots+A_{k j} a_{k}=0$ for all $j \in\{k+1, \ldots, n\}$. Thus $\left(y_{1}, \ldots, y_{n}\right)=$ $(0, \ldots, 0)$. This shows that im $g_{A} \cap U=\{(0, \ldots, 0)\}$.

Therefore the proof is complete.

From Theorem 3.8, we clearly have the next two corollaries.

Corollary 3.9. If $A \in R_{n}(F, k)$ is of the form

$$
\left[\begin{array}{cccccc}
a_{11} & \cdots & a_{1 k} & 0 & \cdots & 0 \\
\vdots & \ddots & \vdots & \vdots & \ddots & \vdots \\
a_{k 1} & \cdots & a_{k k} & 0 & \cdots & 0 \\
0 & \cdots & 0 & 0 & \cdots & 0 \\
\vdots & \ddots & \vdots & \vdots & \ddots & \vdots \\
0 & \cdots & 0 & 0 & \cdots & 0
\end{array}\right]
$$

then $A$ is regular in $R_{n}(F, k)$.

Corollary 3.10. Let $k<n$ and $A \in R_{n}(F, k)$ be of the form

$$
\left[\begin{array}{cccccc}
0 & \cdots & 0 & a_{1, k+1} & \cdots & a_{1, n} \\
\vdots & \ddots & \vdots & \vdots & \ddots & \vdots \\
0 & \cdots & 0 & a_{k, k+1} & \cdots & a_{k n} \\
0 & \cdots & 0 & 0 & \cdots & 0 \\
\vdots & \ddots & \vdots & \vdots & \ddots & \vdots \\
0 & \cdots & 0 & 0 & \cdots & 0
\end{array}\right] .
$$

Then $A$ is regular in $R_{n}(F, k)$ if and only if $A$ is a zero matrix. 
Corollary 3.11. The semigroup $R_{n}(F, k)$ is a regular semigroup if and only if $k=n$.

Proof. If $k<n$, then by Corollary 3.10,

$$
A=\left[\begin{array}{cccc}
0 & 0 & \cdots & 1 \\
0 & 0 & \cdots & 0 \\
\vdots & \vdots & \ddots & 0 \\
0 & 0 & \cdots & 0
\end{array}\right]
$$

is a nonregular element of $R_{n}(F, k)$.

If $k=n$, then $R_{n}(F, k)=M_{n}(F)$. Therefore the converse holds.

Remarks 3.12. In the course of our presentation, we applied Theorem 2.1 and Theorem 2.3 to obtain Theorem 3.4 and Theorem 3.8, respectively. In fact, Theorem 3.4 implies Theorem 3.8 and the converse is also true as a consequence of the following facts:

(i) If the semigroup $S_{1}$ and $S_{2}$ are anti-isomorphic, that is, there is a bijection $\varphi: S_{1} \rightarrow S_{2}$ such that $(x y) \varphi=(y \varphi)(x \varphi)$ for all $x, y \in S_{1}$, it is clear that $\operatorname{Reg}\left(S_{2}\right)=\left(\operatorname{Reg}\left(S_{1}\right)\right) \varphi$.

(ii) The mapping $A \mapsto A^{t}$, the transpose of $A$, from $C_{n}(F, k)\left[R_{n}(F, k)\right]$ into $R_{n}(F, k)\left[C_{n}(F, k)\right]$ is clearly an anti-isomorphism.

Example 3.13. Let $\mathbb{R}$ be the field of real numbers,

$$
A=\left[\begin{array}{lll}
1 & 1 & 0 \\
0 & 0 & 0 \\
0 & 2 & 0
\end{array}\right] \text { and } B=\left[\begin{array}{llll}
0 & 1 & 1 & 0 \\
3 & 2 & 1 & 0 \\
0 & 0 & 0 & 0 \\
0 & 0 & 0 & 0
\end{array}\right]
$$

If we consider $A \in C_{3}(\mathbb{R}, 2)$, then $A$ is not a regular element of $C_{3}(\mathbb{R}, 2)$ by Theorem 3.4 since $A_{11}(1)+A_{12}(-1)=0=A_{21}(1)+A_{22}(-1)$ and $A_{31}(1)+$ $A_{32}(-1)=-2 \neq 0$. Consider $B$ as an element of $C_{4}(\mathbb{R}, 3)$ and $R_{4}(\mathbb{R}, 2)$. By Corollary $3.5, B \in \operatorname{Reg}\left(C_{4}(\mathbb{R}, 3)\right)$. To show that $B \in \operatorname{Reg}\left(R_{4}(\mathbb{R}, 2)\right)$ by Theorem 3.8, let $x_{1}, x_{2} \in \mathbb{R}$ be such that $B_{11} x_{1}+B_{21} x_{2}=0=B_{12} x_{1}+B_{22} x_{2}$. Then $3 x_{2}=0=x_{1}+2 x_{2}$ which implies that $x_{1}=x_{2}=0$, so $B_{13} x_{1}+B_{23} x_{2}=$ $0=B_{14} x_{1}+B_{24} x_{2}$.

\section{References}

[1] I. N. Herstein, Topics in Algebra, Xerox Clooege Publishing, Massachusetts, 1964. 
[2] P. M. Higgins, Techniques of Semigroup Theory, Oxford University Press, New York, 1992.

[3] J. M. Howie, Fundamentals of Semigroup Theory, Clarendon Press, Oxford, 1995.

[4] T. W. Hungerford, Algebra, Springer-Verlag, New York, 1984.

[5] A. I. Malcev, Symmetric groupoids, Math. Sb. (N. S.) 13(73)(1952), 136151.

[6] S. Nenthein, P. Youngkhong and Y. Kemprasit, Regular elements of some transformation semigroups, PU. M. A. - Alg\& Theoretical Comp. Sc., to appear.

[7] J. S. V. Symons, Some results concerning a transformation semigroup, J. Austral. Math. Soc. 19 (Series A)(1975), 413-425.

Received: June 7, 2006 